AperTO - Archivio Istituzionale Open Access dell'Università di Torino

\title{
Big Bang 6Li nucleosynthesis studied deep underground (LUNA collaboration)
}

\section{This is the author's manuscript}

Original Citation:

\section{Availability:}

This version is available http://hdl.handle.net/2318/1625379

since 2017-02-21T12:04:29Z

Published version:

DOI:10.1016/j.astropartphys.2017.01.007

Terms of use:

Open Access

Anyone can freely access the full text of works made available as "Open Access". Works made available under a Creative Commons license can be used according to the terms and conditions of said license. Use of all other works requires consent of the right holder (author or publisher) if not exempted from copyright protection by the applicable law. 


\title{
Big Bang ${ }^{6} \mathrm{Li}$ nucleosynthesis studied deep underground (LUNA collaboration)
}

\author{
D. Trezzi ${ }^{\mathrm{a}}$, M. Anders ${ }^{\mathrm{b}, \mathrm{c}, 1}$, M. Aliottad ${ }^{\mathrm{d}}$, A. Bellini $^{\mathrm{e}}$, D. Bemmerer ${ }^{\mathrm{b}}$, A. Boeltzig $^{\mathrm{f}, \mathrm{g}}$, C. Broggini $^{\mathrm{h}}$, \\ C.G. Bruno ${ }^{\mathrm{d}}$, A. Caciolli ${ }^{\mathrm{h}, \mathrm{i}}$, F. Cavanna ${ }^{\mathrm{e}}$, P. Corvisiero ${ }^{\mathrm{e}}$, H. Costantini $^{\mathrm{e}, 2}$, T. Davinson ${ }^{\mathrm{d}}$, \\ R. Depalo ${ }^{h, i}$, Z. Elekes ${ }^{b}$, M. Erhard ${ }^{\text {h }}$, F. Ferraro ${ }^{\mathrm{e}}$, A. Formicola ${ }^{\mathrm{f}}$, Zs. Fülop ${ }^{\mathrm{j}}$, G. Gervino ${ }^{\mathrm{k}}$, \\ A. Guglielmetti ${ }^{a}$, C. Gustavino ${ }^{l, *}$, Gy. Gyürky ${ }^{\mathrm{j}}$, M. Junker ${ }^{\mathrm{f}}$, A. Lemut ${ }^{\mathrm{e}, 3}$, M. Marta ${ }^{\mathrm{b}, 4}$, \\ C. Mazzocchi $^{\mathrm{a}, 5}$, R. Menegazzo $^{\mathrm{h}}$, V. Mossa ${ }^{\mathrm{m}}$, F. Pantaleo $^{\mathrm{m}}$, P. Prati $^{\mathrm{e}}$, C. Rossi Alvarez $^{\mathrm{h}}$, \\ D.A. Scott ${ }^{d}$, E. Somorjai ${ }^{j}$, O. Straniero ${ }^{n, 0}$, T. Szücs $^{j}$, M. Takacs ${ }^{b}$
}

\footnotetext{
a Università degli Studi di Milano and INFN, Sezione di Milano, Via Celoria 16, 20133 Milano, Italy

${ }^{\mathrm{b}}$ Helmholtz-Zentrum Dresden-Rossendorf, Bautzner Landstr. 400, 01328 Dresden, Germany

${ }^{\mathrm{c}}$ Technische Universität Dresden, Mommsenstrasse 9, 01069 Dresden, Germany

d SUPA, School of Physics and Astronomy, University of Edinburgh, EH9 3JZ Edinburgh, United Kingdom

e Università degli Studi di Genova and INFN, Sezione di Genova, Via Dodecaneso 33, 16146 Genova, Italy

${ }^{\mathrm{f}}$ Laboratori Nazionali del Gran Sasso (LNGS), Via Acitelli 22, 67010 Assergi (AQ), Italy

${ }^{g}$ Gran Sasso Science Institute (GSSI), Viale F. Crispi 7, 67100 L'Aquila (AQ), Italy

h INFN, Sezione di Padova, Via F. Marzolo 8, 35131 Padova, Italy

i Dipartimento di Fisica e Astronomia, Università degli studi di Padova, Via F. Marzolo 8, 35131 Padova, Italy

${ }^{\mathrm{j}}$ Institute for Nuclear Research (MTA ATOMKI), PO Box 51, HU-4001 Debrecen, Hungary

${ }^{\mathrm{k}}$ Università degli Studi di Torino and INFN, Sezione di Torino, Via P. Giuria 1, 10125 Torino, Italy

${ }^{1}$ INFN, Sezione di Roma Sapienza, Piazzale A. Moro 2, 00185 Roma, Italy

m Dipartimento Interateneo, Università degli Studi di Bari and INFN, Sezione di Bari, Via E. Orabona 4, 70125 Bari, Italy

${ }^{n}$ Osservatorio Astronomico di Collurania, Via M. Maggini, 64100 Teramo, Italy

${ }^{\circ}$ INFN, Sezione di Napoli, Via Cintia, 80126 Napoli, Italy
}

\section{A R T I C L E I N F O}

\section{Article history:}

Received 20 November 2015

Revised 2 December 2016

Accepted 22 January 2017

Available online 23 January 2017

\section{Keywords:}

Nuclear Astrophysics

Big Bang Nucleosynthesis

6Li abundance

Underground

\begin{abstract}
A B S T R A C T
The correct prediction of the abundances of the light nuclides produced during the epoch of Big Bang Nucleosynthesis (BBN) is one of the main topics of modern cosmology. For many of the nuclear reactions that are relevant for this epoch, direct experimental cross section data are available, ushering the so-called "age of precision". The present work addresses an exception to this current status: the ${ }^{2} \mathrm{H}(\alpha, \gamma){ }^{6} \mathrm{Li}$ reaction that controls ${ }^{6} \mathrm{Li}$ production in the Big Bang. Recent controversial observations of ${ }^{6} \mathrm{Li}$ in metal-poor stars have heightened the interest in understanding primordial ${ }^{6} \mathrm{Li}$ production. If confirmed, these observations would lead to a second cosmological lithium problem, in addition to the well-known ${ }^{7} \mathrm{Li}$ problem. In the present work, the direct experimental cross section data on ${ }^{2} \mathrm{H}(\alpha, \gamma){ }^{6} \mathrm{Li}$ in the BBN energy range are reported. The measurement has been performed deep underground at the LUNA (Laboratory for Underground Nuclear Astrophysics) $400 \mathrm{kV}$ accelerator in the Laboratori Nazionali del Gran Sasso, Italy. The cross section has been directly measured at the energies of interest for Big Bang Nucleosynthesis for the first time, at $E_{c m}=80,93,120$, and $133 \mathrm{keV}$. Based on the new data, the ${ }^{2} \mathrm{H}(\alpha, \gamma)^{6} \mathrm{Li}$ thermonuclear reaction rate has been derived. Our rate is even lower than previously reported, thus increasing the discrepancy between predicted Big Bang ${ }^{6} \mathrm{Li}$ abundance and the amount of primordial ${ }^{6} \mathrm{Li}$ inferred from observations.
\end{abstract}

(c) 2017 Elsevier B.V. All rights reserved.

\footnotetext{
* Corresponding author.

E-mail address: carlo.gustavino@roma1.infn.it (C. Gustavino).

1 Present address: Sächsisches Staatministerium für Umwelt und Landwirtschaft, Dresden, Germany.

2 Present address: CPPM, Université d'Aix-Marseille, CNRS/IN2P3, Marseille, France.

${ }^{3}$ Deceased.

${ }^{4}$ Present address: GSI, Darmstadt, Germany.

${ }^{5}$ Present address: University of Warsaw, Warsaw, Poland.
} 


\section{Introduction}

Big Bang Nucleosynthesis (BBN) may be used to probe cosmological models and parameters. To this end, abundance predictions from BBN have to be compared with the abundances inferred by astronomers observing the emission and absorption lines in specific astrophysical environments. The pillars of BBN calculations are: Standard Cosmological Big Bang model, the Standard Model of Particle Physics, and the nuclear cross sections of the processes involved in the BBN reaction network. The agreement between calculations and observations for primordial deuterium and ${ }^{4} \mathrm{He}$ (see for example [1]) places cosmology, nuclear and particle physics in a uniquely consistent framework. However, the situation is not as favorable for ${ }^{7} \mathrm{Li}$ and ${ }^{6} \mathrm{Li}$ [2]. For ${ }^{7} \mathrm{Li}$, the predicted abundance is about a factor of 3 higher than the observed one. As of today a clear solution to this puzzle, "the lithium problem", has not been found. Even more complex is the case of ${ }^{6} \mathrm{Li}$, where the predicted abundance is up to three orders of magnitude lower than that inferred from direct observations (the so called "second lithium problem") $[3,4]$.

The aim of the present work is to put the nuclear physics of ${ }^{6} \mathrm{Li}$ production in standard Big Bang scenarios on solid experimental ground. The nuclear cross section of the leading process in the ${ }^{6} \mathrm{Li}$ primordial production, i.e. the ${ }^{2} \mathrm{H}(\alpha, \gamma)^{6} \mathrm{Li}$ fusion reaction, has thus been directly measured in the BBN energy range at LUNA (Laboratory for Underground Nuclear Astrophysics). The measurement has been carried out with the world's only underground accelerator for nuclear astrophysics, situated at the Laboratori Nazionali del Gran Sasso (LNGS), Italy. The data at center-of-mass energy $E$ $=133$ and $93 \mathrm{keV}$ have been previously published in abbreviated form [5]. Here, two new data points at $E=120$ and $80 \mathrm{keV}$ are presented.

The present work is organized as follows: In Sections 2 and 3 a description of astronomical observations and nuclear processes involved in the ${ }^{6} \mathrm{Li}$ production and destruction are given. In Section 4 a short description of the LUNA apparatus is reported (more details in [6]). In Section 5 data analysis is described and finally in Sections 6 and 7 the ${ }^{2} \mathrm{H}(\alpha, \gamma)^{6} \mathrm{Li}$ reaction rate including the new LUNA data points and the resulting conclusions are given.

\section{Review of astronomical data on ${ }^{6} \mathbf{L i}$}

${ }^{6} \mathrm{Li}$ is mainly produced during the BBN epoch and, in more recent epochs, by cosmic ray spallation [7]. For this reason, its primordial abundance is inferred from observations of the atmospheres of hot metal-poor stars in the galactic halo (either main sequence dwarfs or subgiants near the turn-off point). The primordial abundance is then obtained by extrapolating the abundance at zero metallicity. The strength of the lithium absorption line $(\lambda=$ $670.7 \mathrm{~nm}$ ) provides the lithium abundance. As the absorption line of ${ }^{6} \mathrm{Li}$ is slightly shifted towards a higher wavelength compared to ${ }^{7} \mathrm{Li}$, the abundance of ${ }^{6} \mathrm{Li}$ isotope can be derived through the shape analysis of the lithium absorption line. The shape of the absorption line is also affected by the convective motions of the stellar atmosphere thus analysis of the lithium absorption line depends on the theoretical model for the stellar atmosphere.

Recently, Steffen et al. [10] and Lind et al. [11] have pointed out that, by using three-dimensional model atmospheres and dropping the assumption of Local Thermodynamic Equilibrium (3D NLTE), many of the ${ }^{6} \mathrm{Li}$ detections may become non-significant. However, the situation is still unclear. As an example, 3D-NLTE analysis of star HD 84937 provides contradictory results according to different authors [10,11]. In other cases, such as star G020-024, we have a good agreement between one dimensional [3] and three dimensional models [10], respectively. The results obtained in metal-poor
Table 1

Astronomical observations. Relative ${ }^{6} \mathrm{Li} /{ }^{7} \mathrm{Li}$ isotopic abundance in some metal-poor stars obtained using different stellar atmosphere models, in one (1D) or three (3D) dimensions, with or without Local Thermodynamic Equilibrium (LTE, NLTE), respectively.

\begin{tabular}{ccll}
\hline Star name & ${ }^{6} \mathrm{Li} /{ }^{7} \mathrm{Li}[\%]$ & method & ref. \\
\hline HD 84937 & $5.0 \pm 2.0$ & 1D LTE & {$[8]$} \\
& $5.2 \pm 1.9$ & 1D LTE & {$[9]$} \\
& $6.6 \pm 2.4$ & 1D LTE & {$[10]$} \\
& $5.1 \pm 1.5$ & 1D NLTE & {$[4]$} \\
& $5.1 \pm 2.3$ & 3D NLTE & {$[10]$} \\
HD 160617 & $1.1 \pm 1.0$ & 3D NLTE & {$[11]$} \\
& $0.1 \pm 1.2$ & 1D LTE & {$[10]$} \\
& $0.3 \pm 1.2$ & 1D LTE & {$[10]$} \\
& $3.6 \pm 1.0$ & 1D LTE/NLTE & {$[3]$} \\
& $-0.5 \pm 1.2$ & 3D NLTE & {$[10]$} \\
& $-1.3 \pm 1.2$ & 3D NLTE & {$[10]$} \\
G064-012 & $-0.01 \pm 0.01$ & 3D NLTE & {$[12]$} \\
& $5.9 \pm 2.1$ & 1D NLTE & {$[4]$} \\
& $2.3 \pm 2.9$ & 1D LTE & {$[10]$} \\
& $2.0 \pm 2.9$ & 1D LTE & {$[10]$} \\
& $1.2 \pm 2.8$ & 3D NLTE & {$[10]$} \\
& $0.8 \pm 2.7$ & 3D NLTE & {$[10]$} \\
& $0.5 \pm 2.3$ & 3D NLTE & {$[11]$} \\
G020-024 & $11.7 \pm 2.5$ & 1D LTE & {$[10]$} \\
& $7.0 \pm 1.7$ & 1D LTE & {$[3]$} \\
& $9.9 \pm 2.4$ & 3D LTE & {$[10]$} \\
\hline
\end{tabular}

stars (HD 84937, HD 160617, G064-012 and G020-024) where different analyses have been reported in the literature, are shown in Table 1.

In conclusion, although several authors agree on the fact that ${ }^{6}$ Li has been detected on a few halo stars $[2,10]$, the determination of primordial ${ }^{6} \mathrm{Li}$ abundance is still a difficult task, because observations are affected by systematic uncertainties related to the convective motion of the stellar atmosphere. As a consequence, observations with spectrometers with higher sensitivity and resolution would be necessary. At the same time, it would be important to improve stellar modeling in order to firmly establish the primordial abundance of ${ }^{6} \mathrm{Li}$ inferred from observations.

\section{The nuclear physics of primordial ${ }^{6} \mathrm{Li}$}

In the standard BBN framework, the primordial ${ }^{6} \mathrm{Li}$ abundance is mainly determined by two nuclear reactions [13]: the ${ }^{2} \mathrm{H}(\alpha, \gamma)^{6} \mathrm{Li}$ reaction that produces ${ }^{6} \mathrm{Li}$ and the ${ }^{6} \mathrm{Li}(\mathrm{p}, \alpha)^{3} \mathrm{He}$ that destroys it. The ${ }^{6} \mathrm{Li}(\mathrm{p}, \alpha)^{3} \mathrm{He}$ reaction rate is fairly well known in the BBN energy range [14]. On the other hand, the lack of direct measurements at low energy of the ${ }^{2} \mathrm{H}(\alpha, \gamma){ }^{6} \mathrm{Li}$ process makes its reaction rate largely uncertain.

The ${ }^{2} \mathrm{H}(\alpha, \gamma){ }^{6} \mathrm{Li}$ cross section at energies less than $1 \mathrm{MeV}$ is dominated by radiative E2 capture from d waves in the scattering state into the ground state of ${ }^{6} \mathrm{Li}$ through a $3^{+}$resonance at $\mathrm{E}_{R}=$ $711 \mathrm{keV}$. At energies lower than $300 \mathrm{keV}$ in the center-of-mass system system, due to the different angular momentum barriers for $\mathrm{p}$ and $\mathrm{d}$ waves, the E1 contribution is expected to be comparable to the E2 one [15]. For a detailed discussion of theoretical predictions, see Refs. $[15,16]$.

The ${ }^{2} \mathrm{H}(\alpha, \gamma)^{6} \mathrm{Li}$ cross section has never been directly measured in the BBN region of interest ( $30 \lesssim E \lesssim 300 \mathrm{keV}$ ), before the LUNA data first reported in [5]. It is worth nothing, only one attempt to directly measure the ${ }^{2} \mathrm{H}(\alpha, \gamma)^{6} \mathrm{Li}$ cross section at low energy is reported in literature, providing only an upper limit [17]. The other direct measurements have been performed at higher energies, i.e. around the $711 \mathrm{keV}$ resonance [18], and in the $\mathrm{MeV}$ region [19]. In order to unfold the ${ }^{2} \mathrm{H}(\alpha, \gamma){ }^{6} \mathrm{Li}$ cross section at low energy, two different Coulomb dissociation experiments have been performed at 26 [20] and $150 \mathrm{MeV} /$ nucleon [15] projectile energy, respectively. 


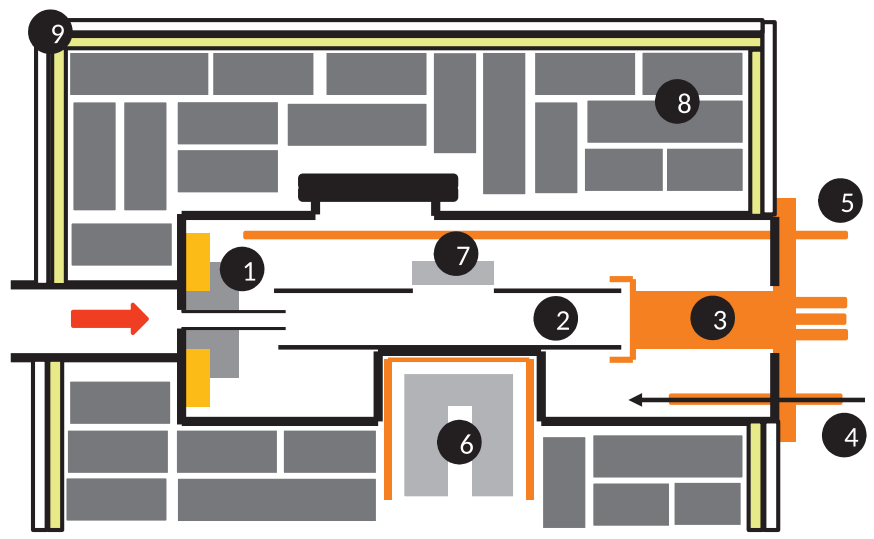

Fig. 1. Experimental setup. The ${ }^{4} \mathrm{He}^{+}$beam enters in the gas target through the collimator (1), passes through the steel pipe (2) and reaches the calorimeter (3). The deuterium inlet (4) is below the calorimeter. The pressure is kept constant by means of a feedback system controlled by a pressure gauge (5). The $\gamma$-rays produced by the ${ }^{2} \mathrm{H}(\alpha, \gamma)^{6} \mathrm{Li}$ reaction are detected by a HPGe detector (6). The beam induced background (see Section 4.2 for details), is monitored by a silicon detector (7). To reduce the environmental background, a lead castle (8) and an anti-radon box (9) surround the detector and gas target.

This technique is mainly sensitive to the electric quadrupole (E2) component of the cross section. However, the competing nuclear breakup process dominates at both beam energies $[15,20]$. Therefore, these data may be interpreted as upper limits of the E2 contribution to the cross section. In conclusion, the ${ }^{6} \mathrm{Li}$ abundance predicted by the BBN theory is affected by large uncertainties because of the lack of data for the ${ }^{2} H(\alpha, \gamma){ }^{6} \mathrm{Li}$ reaction. In this context, the LUNA measurement discussed in this paper represents a significant step forward, providing the first direct measurement of this reaction well inside the BBN energy region.

\section{Experimental setup}

\subsection{Apparatus description}

The measurement was performed by detecting the prompt $\gamma$ rays emitted from the ${ }^{2} \mathrm{H}(\alpha, \gamma)^{6} \mathrm{Li}$ reaction, using the experimental setup shown in Fig. 1. A ${ }^{4} \mathrm{He}^{+}$beam generated by the $400 \mathrm{keV}$ LUNA II accelerator [21] passed several collimators along a powerful gas pumping system to enter the target chamber, in which a deuterium gas pressure of 0.3 mbar was kept by a feedback system. Together with the good energy stability of the accelerator, the windowless gas target (length $17.7 \mathrm{~cm}$ ) allows for an accurate calculation of the beam energy along the entire beam path. The stopping power inside the gas target is quite low $(0.18 \mathrm{keV} / \mathrm{cm}$ at $400 \mathrm{keV}$ beam energy). The beam intensity (typically $0.3 \mathrm{~mA}$ ) was measured by a beam calorimeter with a constant temperature gradient.

The target chamber and HPGe detector were shielded by at least $20 \mathrm{~cm}$ of lead in all directions and the setup was enclosed in an anti-radon box flushed with nitrogen to ensure a very low and stable natural background (see Fig. 2). The $\gamma$-rays were detected by a large (137\% relative efficiency) high-purity germanium (HPGe) detector placed at a $90^{\circ}$ angle with respect to the ion beam direction in a close geometry, to reach a full-energy peak detection efficiency of $1.7 \%$ at $1.6 \mathrm{MeV}$. An energy resolution of $2.8 \mathrm{keV}$ at 1.6 MeV $\gamma$-ray energy was achieved. A more detailed description of the setup can be found in $[6,22]$.

\subsection{Neutron induced background}

${ }^{2} \mathrm{H}(\alpha, \gamma){ }^{6} \mathrm{Li}$ is not the only reaction that occurred in the gas target. Deuterons can be elastically scattered by the incoming alpha

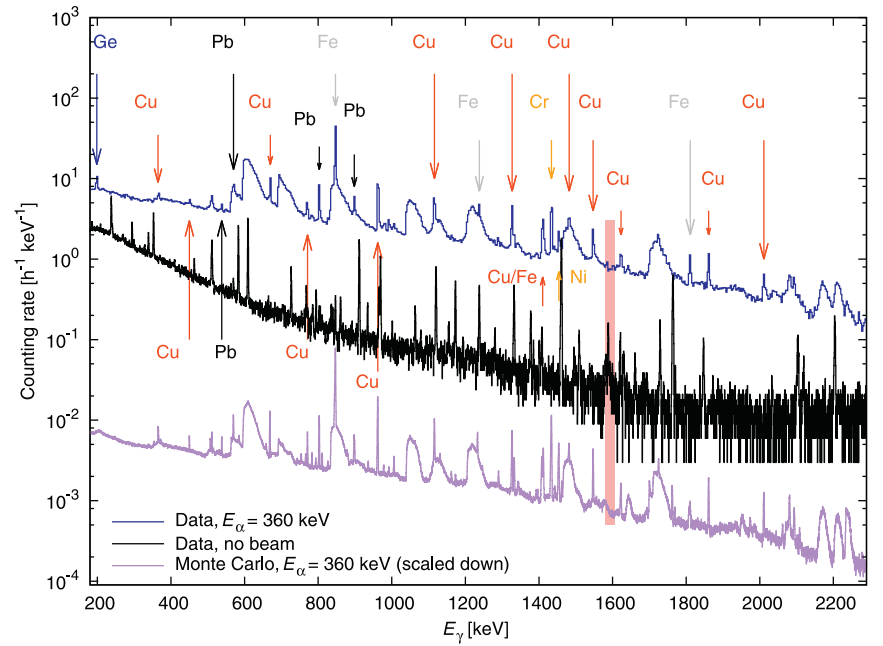

Fig. 2. Black line: Natural background measured with a HPGe detector. Blue line: The ${ }^{2} \mathrm{H}(\alpha, \gamma){ }^{6} \mathrm{Li}$ spectrum at $\mathrm{E}_{\alpha}=360 \mathrm{keV}$ and $\mathrm{p}_{\text {target }}=0.3 \mathrm{mbar}$ (natural background has been subtracted). Violet line: The ${ }^{2} \mathrm{H}(\alpha, \gamma)^{6} \mathrm{Li}$ simulated spectrum at $\mathrm{E}_{\alpha}$ $=360 \mathrm{keV}$ and $\mathrm{p}_{\text {target }}=0.3 \mathrm{mbar}$ (scaled down). The arrows indicate the $\left(\mathrm{n}, \mathrm{n}^{\prime} \gamma\right)$ lines. The triangular peaks are due to the interaction of neutrons with the germanium (see text). More details are reported in [6]. The red band indicates the ${ }^{2} \mathrm{H}(\alpha, \gamma){ }^{6} \mathrm{Li}$ region of interest at $\mathrm{E}_{\alpha}=360 \mathrm{keV}$. The measured beam induced background at LUNA is about one order of magnitude less than the natural background expected on Earth surface experiments with the same setup [24]. (For interpretation of the references to colour in this figure legend, the reader is referred to the web version of this article.)

particles, receiving a kinetic energy that enables them to induce the ${ }^{2} \mathrm{H}\left({ }^{2} \mathrm{H}, \mathrm{p}\right){ }^{3} \mathrm{H}$ and ${ }^{2} \mathrm{H}\left({ }^{2} \mathrm{H}, \mathrm{n}\right)^{3} \mathrm{He}$ reactions with the surrounding deuterons in the gas. Protons from the ${ }^{2} \mathrm{H}\left({ }^{2} \mathrm{H}, \mathrm{p}\right)^{3} \mathrm{H}$ reaction were monitored with a silicon detector (see Fig. 1 and [6] for details). Neutrons from the ${ }^{2} \mathrm{H}\left({ }^{2} \mathrm{H}, \mathrm{n}\right)^{3} \mathrm{He}$ reaction can interact with the surrounding materials (iron, copper, lead and germanium) producing $\gamma$-rays through $\left(n, n^{\prime} \gamma\right)$ reactions. In order to reduce the deuteron scattering probability, a pipe with a square section of $2 \mathrm{~cm}$ was installed along the beam line. This pipe limits the free path of the elastically scattered deuterons inside the gas, thus reducing the number of neutrons produced by the ${ }^{2} \mathrm{H}\left({ }^{2} \mathrm{H}, \mathrm{n}\right){ }^{3} \mathrm{He}$ reaction to a value of about 10 neutrons per second. The rate of this neutron induced background $\mathrm{BG}^{\text {neutron }}$ in the Region of Interest (RoI) for the ${ }^{2} \mathrm{H}(\alpha, \gamma){ }^{6} \mathrm{Li}$ reaction is about one order of magnitude higher than the expected signal but nevertheless much lower than the usual background present on Earth's surface experiments [23].

Fig. 2 shows the in-beam $\mathrm{E}_{\alpha}=360 \mathrm{keV} \gamma$-ray spectrum as well as a comparative background spectrum. It contains many lines and several triangular shaped peaks (for more details see [6]). The formers are due to the natural background and $\left(n, n^{\prime} \gamma\right)$ beam induced reactions on the materials surrounding the reaction chamber. The triangular shaped peaks are due to $\left(n, n^{\prime} \gamma\right)$ beam induced reactions of energetic neutrons on the Germanium detector itself. The triangular shape arises from the germanium nucleus recoil energy deposited in the detector. Similar features have been observed at all beam energies investigated. The neutron induced background spectrum as a function of the beam energy has been studied with a dedicated simulation, in which our experimental setup has been reproduced. The simulated spectra are consistent with the experimental ones [22]. The main difference between the spectra obtained at different beam energies (experimental and simulated), is an overall energy dependence of the HPGe response at $E_{\gamma} \gtrsim$ $1500 \mathrm{keV}$, in such a way the bin-by-bin ratio produced at different beam energies is not constant but weakly depends on the beam energy. For what concerns the complex spectral structure, the most prominent difference between spectra at different beam 


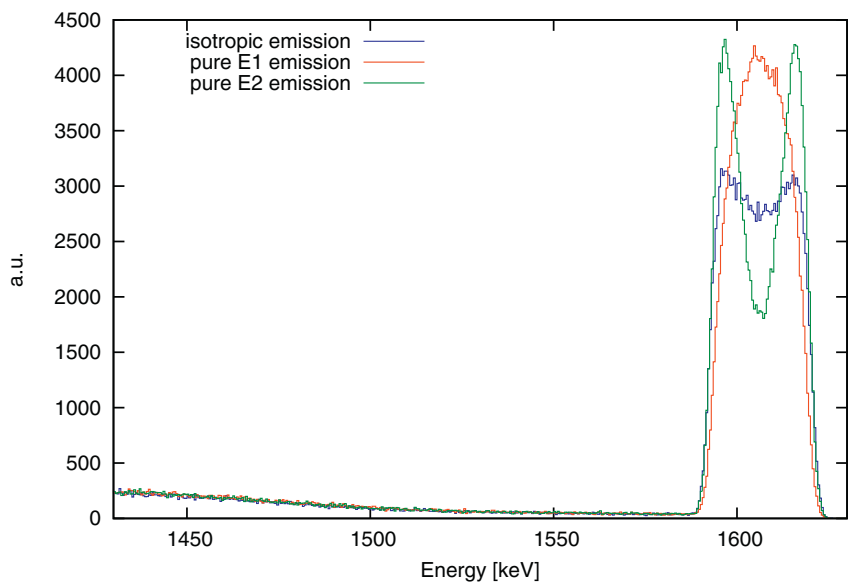

Fig. 3. Simulated spectra of the ${ }^{2} \mathrm{H}(\alpha, \gamma){ }^{6} \mathrm{Li}$ reaction $\left(\mathrm{E}_{\alpha}=400 \mathrm{keV}\right)$, assuming different angular distribution for the emitted photons. Note that the RoI does not depends on the adopted angular distributions.

energies is related to the excitation of ${ }^{56} \mathrm{Fe}$ nuclei to the $2658 \mathrm{keV}$ state, which decays in a cascade via emission of $1811 \mathrm{keV}$ and $847 \mathrm{keV} \gamma$-rays. The strength of the gamma lines associated to the $2658 \mathrm{keV}$ state increases with the beam energy. In fact, at high energy ( 360 or $400 \mathrm{keV}$ ) there are more neutrons available to populate the $2658 \mathrm{keV}$ level with respect to low beam energies (280 or $240 \mathrm{keV}$ ), thus explaining the increase of the $1811 \mathrm{keV}$ counting rate with the beam energy. This effect is confirmed in a dedicated simulation in which the Rutherford $\alpha\left({ }^{2} \mathrm{H},{ }^{2} \mathrm{H}\right) \alpha$ scattering and the ${ }^{2} \mathrm{H}\left({ }^{2} \mathrm{H}, \mathrm{n}\right)^{3} \mathrm{He}$ reaction are implemented. As expected, it has been found that the doppler effect determines a broadening of neutron energy distribution with the beam energy, in agreement with the observed effect on the ${ }^{56} \mathrm{Fe}$ gamma lines $[6,22]$.

\section{Data analysis}

\subsection{General approach}

In this section the data analysis of the ${ }^{2} \mathrm{H}(\alpha, \gamma)^{6} \mathrm{Li}$ reaction at beam energies $240,280,360$ and $400 \mathrm{keV}$ is reported. The energy of photons produced in the ${ }^{2} \mathrm{H}(\alpha, \gamma){ }^{6} \mathrm{Li}$ reaction depends on the beam energy and on the doppler effect. It can be expressed by the following relativistic formula (in which $c=\hbar=1$ ):

$E_{\gamma}=\frac{m_{H e}^{2}+m_{d}^{2}-m_{L i}^{2}+2 m_{d}\left(E_{\alpha}+m_{H e}\right)}{2\left[E_{\alpha}+m_{H e}+m_{d}-p_{H e} \cos \left(\theta_{l a b}\right)\right]}$

In this equation $E_{\gamma}$ is the photon energy, $\mathrm{m}_{H e}, \mathrm{~m}_{d}$ and $\mathrm{m}_{L i}$ are the masses of nuclides involved in the reaction, $p_{H e}=$ $\sqrt{E_{\alpha}\left(E_{\alpha}+2 m_{H e}\right)}$ is the $\alpha$ particle momentum and $\theta_{\text {lab }}$ is the angle of emitted photon with respect to the beam axis.

This formula indicates that fully detected photons populate a well defined region of interest (RoI) for each beam energy. The energy range of the RoIs only depends on the beam energy and on the angular acceptance of the HPGe detector $\left(10^{\circ} \lesssim \theta_{\text {lab }} \lesssim\right.$ $170^{\circ}$ ). It has been determined with the GEANT Monte Carlo simulation, in which the experimental conditions are reproduced and the Eq. (1) is implemented. The angular distribution of photons produced by the ${ }^{2} \mathrm{H}(\alpha, \gamma){ }^{6} \mathrm{Li}$ only affects the distribution of fully detected photons inside the RoI. Fig. 3 shows the simulated energy spectra for different angular distribution of emitted photons. The fully detected photons produced by the ${ }^{2} \mathrm{H}(\alpha, \gamma)^{6} \mathrm{Li}$ reaction have the following RoIs: [1542,1563] keV, [1554,1577] keV, $[1580,1606] \mathrm{keV}$ and $[1592,1620] \mathrm{keV}$, respectively for beam energies of $240,280,360$ and $400 \mathrm{keV}$.
We developed two independent analysis methods. Our data have been grouped in pairs $(i, j)$ of different beam energy runs characterized by non-overlapping RoIs, comparable statistics and similar integrated beam current. If we now indicate with $\mathrm{BG}_{i}^{\text {neutron }}$ and $\mathrm{BG}_{j}^{\text {neutron }}$ the rate of neutron induced background relative to $\mathrm{i}$ and $\mathrm{j}$ energies, we have:

$B G_{i}^{\text {neutron }}=\beta_{i j} B G_{j}^{\text {neutron }}$

As it has been discussed in Section 4.2, the shape of beam induced background weakly depends on the beam energy. In this formula this dependence is included in the $\beta_{i j}$ parameter and will be considered in the following.

Both analysis methods provide cross section values $\sigma(E)$, parameterized by the astrophysical $\mathrm{S}$-factor given by:

$S(E)=\sigma(E) E \exp (72.44 / \sqrt{E})$

where $E(\mathrm{keV})$ is the center-of-mass system energy. The two analysis methods named "Flat regions" and "Minimization" will now be described in detail.

\subsubsection{Method A, based on "flat regions"}

After subtraction of the natural background, there are several energy regions without any visible peak. This purely neutroninduced background is mainly due to the Compton continua of several peaks in the $\gamma$-ray spectrum. Therefore, it is possible to parameterize the ratio of background rates inside such "flat regions" at two beam energies along the entire $\gamma$-ray spectrum. In this way, the ratio of background levels can be calculated anywhere in the $\gamma$-ray spectrum. This allows to properly subtract beam induced backgrounds obtained at two different beam energies using as normalization factor the background ratio. Given that the two Rols do not overlap, The two signals $\mathrm{Y}_{\text {net }}^{\mathrm{E}_{\alpha}=400 \mathrm{keV}}$ (RoI $400 \mathrm{keV}$ ) and $\mathrm{Y}_{\text {net }}^{\mathrm{E}_{\alpha}=280 \mathrm{keV}}(\mathrm{RoI} 280 \mathrm{keV})$, can be written as

$Y_{\text {net }}^{400}(400)=Y^{400}(400)-\beta_{400,280} Y^{400}(280)$

$Y_{\text {net }}^{280}(280)=Y^{280}(280)-\frac{1}{\beta_{400,280}} Y^{280}(400)$

Similar equations can be written for the other two beam energies $\left(\mathrm{E}_{\alpha}=240\right.$ and $\left.360 \mathrm{keV}\right)$. The systematic uncertainty coming from the determination of the normalization factor (here called $\beta_{400,280}^{\mathrm{ROI}}$ and $\beta_{360,240}^{\mathrm{ROI}}$ ) from neighboring "flat regions" is difficult to estimate. Therefore, instead of deriving the systematic error bar of $\beta_{400,280}^{\mathrm{ROI}}$, an approach is chosen that cancels its effects on the signal yield, and thus on the final astrophysical S-factor. It is clear from Eqs. (4) and (5) that $\beta_{400,280}^{\mathrm{ROI}}$ uncertainties affect the two yields in opposite ways: If $\beta_{400,280}^{\mathrm{ROI}}$ is overestimated, $Y_{\text {net }}^{400}(400)$ decreases but $Y_{\text {net }}^{280}(280)$ increases. However, we found that the yield sum $Y_{\text {net }}^{400}(400)+Y_{\text {net }}^{280}(280)$ is independent from $\beta_{400,280}^{\mathrm{ROI}}$, so that the issue of the error estimation of this parameter becomes moot. To extract the astrophysical S-factor at a given energy from the yield sum $Y_{\text {net }}^{400}(400)+Y_{\text {net }}^{280}(280)$, it is necessary to make an assumption on the energy dependence of the astrophysical S-factor inside the LUNA energy range [25]. For the data analysis, various assumptions ranging from $S(400 \mathrm{keV}) / S(280 \mathrm{keV})=1.1-1.5$ have been tested, where 1.1 corresponds to pure E1 and 1.5 to pure E2. The S-factor result does not change outside its statistical error bar in any of the cases tested. The Method A analysis results are shown in Table 2, where the theoretical S-factor trend indicated in [15] is assumed, with a ratio $S(400 \mathrm{keV}) / S(280 \mathrm{keV})=1.3$.

In summary, in method A the effects of the background subtraction cancel out, so the result does not depend on the uncertainty of the background subtraction. However, this method requires a theoretical assumption for the shape of the S-factor curve, and several 
Table 2

Astrophysical S-factor and nuclear cross section. In the table are indicated: the effective center-of-mass energy E [keV], the beam energy $\mathrm{E}_{\alpha}[\mathrm{keV}]$, the deuterium gas target pressure $\mathrm{P}$ [mbar], the measurement time $\mathrm{t}[\mathrm{h}]$, the integrated beam current $\mathrm{Q}[\mathrm{C}]=$ $I_{\text {beam }} t$, the net reaction yield $Y[$ counts/C] and its statistical error (the counting error for the "method A" includes the uncertainty due to the theoretical assumption, see text), the total cross section $\sigma$ [pb] with counting and systematic errors, the astrophysical S-factor S [keV $\mu \mathrm{b}]$ with counting and systematic errors. The results of analysis "Method B" are adopted in this work. The data points at 80 and $93 \mathrm{keV}$ are less than two standard deviations above zero and should be considered as upper limits.

\begin{tabular}{lllllllll}
\hline $\mathrm{E}[\mathrm{keV}]$ & $\mathrm{E}_{\alpha}[\mathrm{keV}]$ & $\mathrm{P}[\mathrm{mbar}]$ & $\mathrm{Q}[\mathrm{C}]$ & $\mathrm{t}[\mathrm{h}]$ & Yield [counts/C] & cross section [pb] & S-factor [keV $\mu \mathrm{b}]$ & $\mathrm{Method}$ \\
\hline 80 & 240 & 0.306 & 211.5 & 217.7 & $0.23 \pm 0.64$ & $9 \pm 25 \pm 1$ & $2.4 \pm 6.7 \pm 0.3$ & $\mathrm{~A}$ \\
93 & 280 & 0.308 & 538.9 & 490.9 & $0.46 \pm 0.46$ & $18 \pm 18 \pm 2$ & $3.0 \pm 3.0 \pm 0.4$ & $\mathrm{~A}$ \\
120 & 360 & 0.306 & 252.7 & 205.2 & $0.94 \mp 0.57$ & $37 \mp 23 \pm 5$ & $3.3 \mp 2.0 \pm 0.4$ & $\mathrm{~A}$ \\
133 & 400 & 0.306 & 514.3 & 437.7 & $1.46 \mp 0.46$ & $58 \mp 18 \pm 8$ & $4.1 \mp 1.3 \pm 0.5$ & $\mathrm{~A}$ \\
80 & 240 & 0.306 & 211.5 & 217.7 & $0.11 \pm 0.51$ & $4 \pm 20 \pm 0.4$ & $1.1 \pm 5.2 \pm 0.1$ & $\mathrm{~B}$ \\
93 & 280 & 0.308 & 538.9 & 490.9 & $0.42 \pm 0.25$ & $16 \pm 10 \pm 2$ & $2.7 \pm 1.6 \pm 0.3$ & $\mathrm{~B}$ \\
120 & 360 & 0.306 & 252.7 & 205.2 & $1.05 \pm 0.47$ & $40 \pm 18 \pm 6$ & $3.6 \pm 1.6 \pm 0.5$ & $\mathrm{~B}$ \\
133 & 400 & 0.306 & 514.3 & 437.7 & $1.50 \pm 0.32$ & $59 \pm 13 \pm 7$ & $4.1 \pm 0.9 \pm 0.5$ & $\mathrm{~B}$ \\
\hline
\end{tabular}

assumptions have been tested. Even still, a radically different Sfactor curve cannot, in principle, be excluded. An alternative analysis method to derive the cross section without any theoretical assumption on the shape of the S-factor curve is described in the next section.

\subsubsection{Method $B$, based on minimization procedures}

This method is based on Eq. (2). The $\gamma$-ray spectrum rate $R_{i}$ is composed by the neutron-induced background $B G_{i}^{\text {neutron }}$, the natural background $B G^{\text {room }}$ and the ${ }^{2} \mathrm{H}(\alpha, \gamma){ }^{6} \mathrm{Li}$ counting rate.

This latter can be expressed as $k_{i} N_{i}$, in which $k_{i}$ is a scaling parameter and $N_{i}$ is the energy distribution due to the photons produced by the ${ }^{2} \mathrm{H}(\alpha, \gamma){ }^{6} \mathrm{Li}$ reaction. The $N_{i}$ distribution has been obtained with the dedicated GEANT simulation in which all the details of the experimental conditions are reproduced and ${ }^{2} \mathrm{H}(\alpha, \gamma){ }^{6} \mathrm{Li}$ reactions are generated along the beam axis (see Fig. 3 ). The $\mathrm{BG}^{\text {neutron }}$ rate can be written as follows:

$B G_{i}^{\text {neutron }}=R_{i}-B G^{\text {room }}-k_{i} N_{i}$

Substituting Eq. (6) in Eq. (2) we obtain:

$R_{i}-B G^{\text {room }}-k_{i} N_{i}-\beta_{i j}\left[R_{j}-B G^{\text {room }}-k_{j} N_{j}\right]=0$

With the "Method B" the free parameters $\beta_{i j}, \mathrm{k}_{i}$ and $\mathrm{k}_{j}$ in the Eq. (7) are obtained with a MINUIT $\chi^{2}$ (least squares) minimization procedure in the energy window $[1500,1625] \mathrm{keV}$, which includes all the considered RoIs.

As stated above, the shape of the distribution of fully detected photons inside a RoI strongly depends on the adopted angular distribution of emitted photons (see Fig. 3). In this concern, all the counting levels inside of the Rol's (natural background, beam induced background and signals) have been averaged before the minimization procedure, to make analysis sensitive only to the total cross section and not dependent on angular distribution assumptions. The HPGe efficiency for different angular distributions has been tested with the simulation. As a result, the efficiency uncertainty due to the unknown angular distribution has been conservatively quoted at the $9 \%$ level.

As discussed in Section 4.2, the $\beta_{i j}$ parameter weakly depends on the energy. This dependence has been modeled with a second order polynomial in our minimization region. This correction determines a negligible variation of the cross sections, about a factor four lower than the statistical errors, for all the four considered data points. As discussed in Section 4.2, the strength of the $1811 \mathrm{keV}$ line slightly depends on the beam energy. Although the line is outside the minimization interval, its Compton edge is at about $1580 \mathrm{keV}$. To evaluate its effect in the analysis results, this line and its Compton edge have been subtracted from the experimental spectra. To do so, the strength of the $1811 \mathrm{keV}$ peak of each dataset has been used to normalize and subtract the simulated spectrum. This correction was found to be negligible as well, about a factor 20 lower than the statistical error, for all the four energies considered. The results of Method B described above are shown in Table 2 . The analysis has been repeated using wider energy windows for the minimization procedure, up to $500<E<$ $2500 \mathrm{keV}$, obtaining results fully consistent with those presented in Table 2. As a consistency check, the minimization procedure has been repeated in energy windows not containing the Rol's. As expected, the counting difference between paired spectra is always consistent with zero, within statistical fluctuations [26].

\section{Results and astrophysical aspects}

\subsection{Astrophysical S-factor and nuclear cross section}

The relevant measurement parameters and the results of the two analysis are reported in Table 2 . The yield $Y_{i}$ inside a RoI is defined by the following formula:

$Y_{i}=k_{i} n_{i}^{R o I} t_{i} / Q_{i}$

where $n_{i}^{\text {RoI }}$ are the net counts/sec in the RoI, $t_{i}$ is the acquisition time. In the "Method $A$ " the error of $Y_{i}$ reported in Table 2 is due to the statistical fluctuations inside the two paired Rol's and to the uncertainties of the theoretical assumption adopted to establish the energy trend of the cross section. The yield uncertainty obtained with "Method B" has been obtained in the $\chi^{2}$ (least squares) minimization procedure of MINUIT, in which the correlation between $\beta, k_{i}$, and $k_{j}$ is taken into account by means of covariance matrix. It is essentially due to the counting fluctuations and therefore it determines the statistical significance of the obtained results. Note that the significance of $Y_{\text {net }}^{400}(400)$ is more than $4 \sigma$, while $Y_{\text {net }}^{240}(240)$ is compatible with zero. As a matter of fact, in the $[1592,1620] \mathrm{keV} \operatorname{RoI}\left(E_{\mathrm{cm}}=133 \mathrm{keV}\right)$ the measured excess is about 750 counts and the background level is 10 times higher for $\mathrm{E}_{\alpha}=400,280 \mathrm{keV}$. On the contrary, the excess in the [1542,1563] $\mathrm{keV} \operatorname{RoI}(\mathrm{Ecm}=80 \mathrm{keV})$ is of only 23 events, a negligible value with respect to the statistical fluctuation of the corresponding background (see Table 2).

For both the analysis the systematic error depends on the uncertainties on the working conditions and represents a possible common bias to all the measurements, without affecting the statistical significance of the obtained results. Table 3 lists the main sources of systematic uncertainties. The largest contribution is due to the unknown angular distribution of the emitted $\gamma$-rays, affecting the percentage of detected photons. The $\gamma$-ray detection efficiency was measured with calibrated ${ }^{137} \mathrm{Cs},{ }^{60} \mathrm{Co}$, and ${ }^{88} \mathrm{Y}$ sources as a function of their position along the beam axis. Due to the close geometry used, the data have been corrected for the truecoincidence summing out effect $[6,22]$.

The reproducibility of the calorimeter calibration was at the $3 \%$ level. This value was adopted as the uncertainty on the beam cur- 
Table 3

Systematic uncertainties and their contribution to the S-factor total budget error.

\begin{tabular}{ll}
\hline Source & Systematic uncertainties \\
\hline Angular distribution & $9 \%$ \\
Detector efficiency & $8 \%$ \\
Beam current & $3 \%$ \\
Temperature & $3 \%$ \\
Pressure & $1 \%$ \\
Target length & $1 \%$ \\
Gas purity & $1 \%$ \\
Beam energy & $<1 \%$ \\
total & $\mathbf{1 3 \%}$ \\
\hline
\end{tabular}

rent determination. The correction on the gas temperature due to the heating of the beam has been estimated by monitoring the temperature in the reaction chamber and from a previous measurement at LUNA for ${ }^{3} \mathrm{He}$ gas, in a setup similar to the present one, by double elastic scattering [36]. In our working conditions, the average temperature of the gas impinged by the beam has been estimated to be $(318 \pm 10) \mathrm{K}[6]$.

The other systematic errors shown in Table 3 are negligible in this concern.

In Fig. 4 (top) the spectra with $\mathrm{E}_{\alpha}=400 \mathrm{keV}$ and $280 \mathrm{keV}$ are compared and the counting excess inside the two RoIs are highlighted. The excess at $\mathrm{E}_{\alpha}=280 \mathrm{keV}$ is less evident with respect to the $\mathrm{E}_{\alpha}=400 \mathrm{keV}$ one because of its lower statistical significance. Fig. 4 (bottom) is relative to the $\mathrm{E}_{\alpha}=360 \mathrm{keV}$ and $240 \mathrm{keV}$ runs. Note that the counting excess of the $\mathrm{E}_{\alpha}=360 \mathrm{keV}$ run is shifted with respect to the $\mathrm{E}_{\alpha}=400 \mathrm{keV}$ one, as expected from kinematics. The S-factor values obtained using the two different analysis methods (Table 2) are mutually consistent. The assumptions considered in the two cases are complementary and provide a robust overall data analysis. The method $\mathrm{B}$ is adopted here because it is based only on the LUNA data, without any theoretical assumption.

Fig. 5 shows the presently obtained cross section compared with literature data and theoretical curves. The LUNA results provide the only direct measurement of the ${ }^{2} \mathrm{H}(\alpha, \gamma)^{6} \mathrm{Li}$ cross section inside the BBN energy window.

\subsection{Thermonuclear reaction rate and astrophysical implications}

In order to compute the thermonuclear reaction rate for the temperatures of interest in BBN, in addition to the present cross section data, some assumptions have to be made on the behavior of the cross section at different energies.

The total cross section is given by the sum of electric dipole (E1) and quadrupole (E2) contributions. The E2 contribution is relatively well-constrained by the direct measurement around the $E$ $=0.711 \mathrm{MeV}$ resonance. Therefore, for the reaction rate determination the E2 contribution given by the latest theoretical work by Hammache and co-workers [15] is adopted here.

The situation is very different for the E1 contribution which is only constrained by the present data, which were not available at the time of publication of recent theoretical curves [15,27]. In order to address this problem, the Hammache E1 curve is rescaled so that the sum of E1 and E2 curves matches the present data. As the E1 component gives only a very small contribution at high energy, this scaling does not affect the good match of theoretical E2 curve and previous, high-energy data (see also Fig. 5).

The thermonuclear reaction rate obtained based on our data is much lower than the previous rate by Caughlan and Fowler (CF88, [28]) but also lower than all other previously reported thermonuclear reaction rates $[15,27,29]$ (see Fig. 6 and Table 4). The relative uncertainty of the present rate is $25 \%$, given by the systematic and statistical errors of the present data, combined in quadrature.

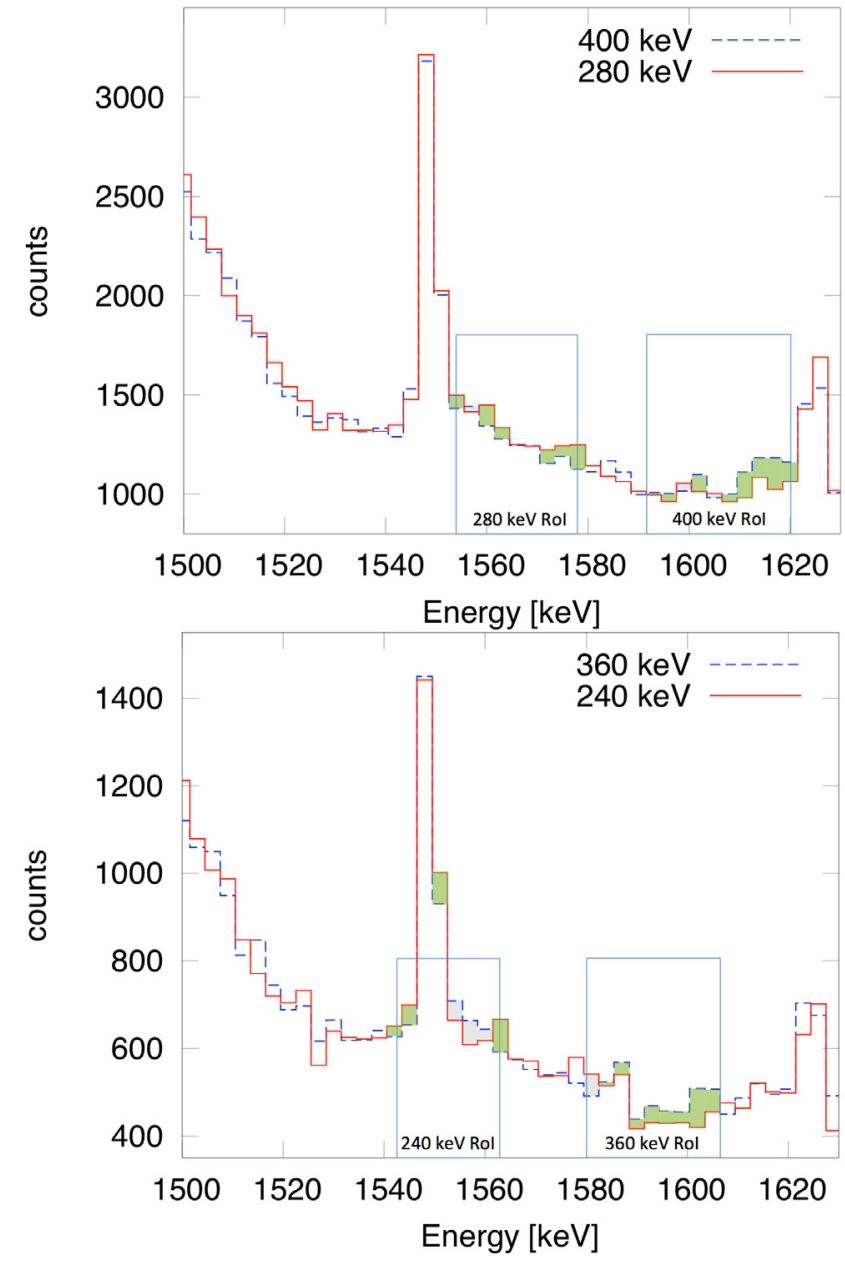

Fig. 4. Top: Experimental spectra for $\mathrm{E}_{\alpha}=400 \mathrm{keV}$ (dashed blue line) and for $\mathrm{E}_{\alpha}=280 \mathrm{keV}$ (full red line). The natural background has been subtracted and the $\mathrm{E}_{\alpha}=280 \mathrm{keV}$ spectrum has been rescaled (see text). Rols at each energy are indicated. The observed counting excess (in green) or defect (in gray) are highlighted bin per bin. See Ref. [5] for a figure displaying the subtracted spectra. Bottom: Same as for the top spectra, but for $\mathrm{E}_{\alpha}=360 \mathrm{keV}$ (dashed blue line) and for the $\mathrm{E}_{\alpha}=$ $240 \mathrm{keV}$ (full red line). Note that the counting excess at $\mathrm{E}_{\alpha}=360 \mathrm{keV}$ is shifted towards lower energy with respect to the $\mathrm{E}_{\alpha}=400 \mathrm{keV}$ one, as foreseen from kinematics. (For interpretation of the references to colour in this figure legend, the reader is referred to the web version of this article.)

The slope as a function of temperature is similar for all the reported works [14,15,27,29 present] for $T_{9}<0.1$, where $T_{9}$ is the temperature expressed in GK.

For computational purposes, the rate can be approximated within $1.5 \%$ by the following analytical formula:

$$
\begin{aligned}
N_{\mathrm{A}}\langle\sigma v\rangle= & 20.070 T_{9}^{-2 / 3} \exp \left(-7.470 / T_{9}^{1 / 3}\right) \\
& {\left[1.000-4.709 T_{9}^{1 / 3}+17.219 T_{9}^{2 / 3}-23.839 T_{9}\right.} \\
& \left.+19.270 T_{9}^{4 / 3}-3.752 T_{9}^{5 / 3}\right]+65.409 T_{9}^{-3 / 2} \\
& \exp \left(-7.565 T_{9}^{-1}\right)
\end{aligned}
$$

The impact of the present new thermonuclear reaction rate on the amount of ${ }^{6} \mathrm{Li}$ produced in BBN was investigated. To this end, a BBN calculation has been performed with the widely used Smith, Kawano, and Malaney (SKM, [30]) code. In the computation, up to date values for the neutron lifetime (880.1 s [31]) and for the final baryon-to-photon ratio $\left(\eta=6.102 \times 10^{-10}[1]\right)$ have been used. The nuclear reaction rates were kept unchanged, except for the presently updated ${ }^{2} \mathrm{H}(\alpha, \gamma){ }^{6} \mathrm{Li}$ rate. The resulting abundance is 


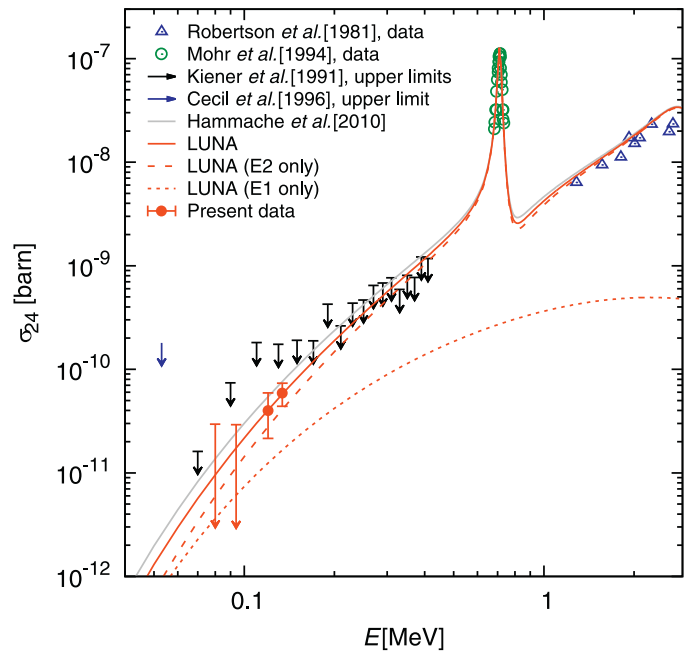

Fig. 5. Cross section of the ${ }^{2} \mathrm{H}(\alpha, \gamma){ }^{6} \mathrm{Li}$ reaction. The LUNA data (method B analysis, see Table 2) are reported in red. The cross section measured at $E_{c m}=80 \mathrm{keV}$ and $E_{c m}=93 \mathrm{keV}$ are shown as upper limits (90\% confidence level) because the experimental results are compatible with zero within two $\sigma \mathrm{s}$. The previous measurements and upper limits are also reported: blue triangles [19], green circle [18], black arrows [20] (upper limits), blue dashed arrow [17] (upper limits). Also shown is our recommended total cross section curve (red full line), together with the adopted E2 component [15] and the E1 component derived from LUNA data (see text). The Hammache et al. [15] total cross section curve is also reported (gray full line). (For interpretation of the references to colour in this figure legend, the reader is referred to the web version of this article.)

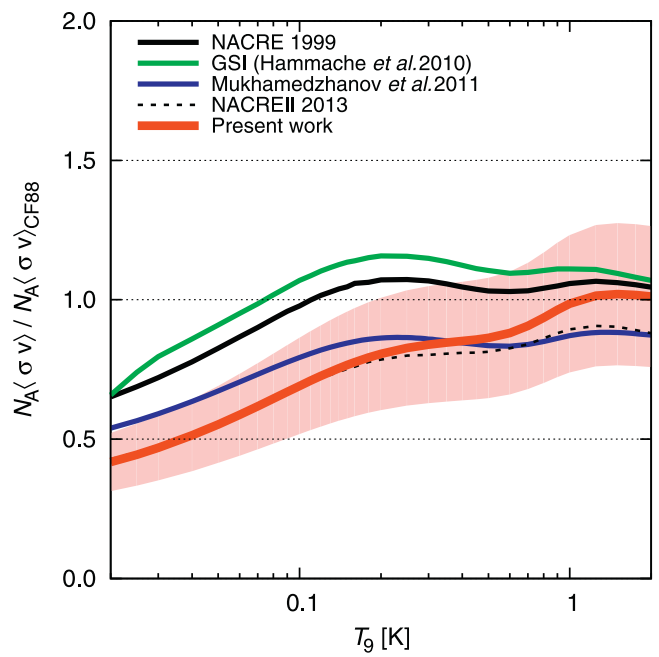

Fig. 6. Ratios of different ${ }^{2} \mathrm{H}(\alpha, \gamma){ }^{6} \mathrm{Li}$ thermonuclear reaction rates to the commonly adopted CF88 [28] rate. NACRE (black dashed curve [29]); Hammache et al. (green short-dashed curve [15]); Mukhamedzhanov et al. (blue dotted curve [27]); NACREII (orange dot-dashed curve [14]). The rate from the present work (red solid line, and shaded error band) is also shown. (For interpretation of the references to colour in this figure legend, the reader is referred to the web version of this article.)

${ }^{6} \mathrm{Li} / \mathrm{H}=(0.80 \pm 0.18) \times 10^{-14}, 27 \%$ lower than the value obtained when using the CF88 [28] rate for ${ }^{2} \mathrm{H}(\alpha, \gamma)^{6} \mathrm{Li}$.

As a further step, also the ${ }^{6} \mathrm{Li} /{ }^{7} \mathrm{Li}$ isotopic ratio from $\mathrm{BBN}$ has been determined, in order to enable a comparison with the observations in metal poor stars that usually report ${ }^{6} \mathrm{Li} /{ }^{7} \mathrm{Li}$ isotopic ratios. For this purpose, ${ }^{3} \mathrm{He}(\alpha, \gamma)^{7} \mathrm{Be}$ reaction rate evaluated by Kontos et al. [32] is used, which is within $1.5 \%$ of the slightly updated de Boer et al. [33] and Takács et al. [34] rates. The excitation function adopted in that work closely tracks the LUNA data on ${ }^{3} \mathrm{He}(\alpha, \gamma){ }^{7} \mathrm{Be}[35,36]$, which are the only recent experimental data on this reaction at energies below $300 \mathrm{keV}$, most relevant for BBN.
Table 4

Thermonuclear reaction rate. Thermonuclear reaction rate for the ${ }^{2} \mathrm{H}(\alpha, \gamma){ }^{6} \mathrm{Li}$ reaction from the present work The relative uncertainty of the present rate is $25 \%$. See text for details.

\begin{tabular}{|c|c|c|c|}
\hline $\begin{array}{l}T_{9} \\
{[\mathrm{~K}]}\end{array}$ & $\begin{array}{l}N_{\mathrm{A}}\langle\sigma v\rangle \\
{\left[\mathrm{cm}^{3} \mathrm{~mol}^{-1} \mathrm{~s}^{-1}\right]}\end{array}$ & $\begin{array}{l}\boldsymbol{T}_{\mathbf{9}} \\
{[\mathrm{K}]}\end{array}$ & $\begin{array}{l}N_{\mathrm{A}}\langle\sigma v\rangle \\
{\left[\mathrm{cm}^{3} \mathrm{~mol}^{-1} \mathrm{~s}^{-1}\right]}\end{array}$ \\
\hline 0.001 & $4.969 \times 10^{-30}$ & 0.090 & $5.227 \times 10^{-06}$ \\
\hline 0.002 & $1.438 \times 10^{-23}$ & 0.100 & $9.047 \times 10^{-06}$ \\
\hline 0.003 & $1.909 \times 10^{-20}$ & 0.110 & $1.464 \times 10^{-05}$ \\
\hline 0.004 & $1.759 \times 10^{-18}$ & 0.120 & $2.246 \times 10^{-05}$ \\
\hline 0.005 & $4.356 \times 10^{-17}$ & 0.130 & $3.297 \times 10^{-05}$ \\
\hline 0.006 & $5.017 \times 10^{-16}$ & 0.140 & $4.669 \times 10^{-05}$ \\
\hline 0.007 & $3.525 \times 10^{-15}$ & 0.150 & $6.411 \times 10^{-05}$ \\
\hline 0.008 & $1.759 \times 10^{-14}$ & 0.160 & $8.577 \times 10^{-05}$ \\
\hline 0.009 & $6.848 \times 10^{-14}$ & 0.180 & $1.440 \times 10^{-04}$ \\
\hline 0.010 & $2.208 \times 10^{-13}$ & 0.200 & $2.257 \times 10^{-04}$ \\
\hline 0.011 & $6.150 \times 10^{-13}$ & 0.250 & $5.617 \times 10^{-04}$ \\
\hline 0.012 & $1.523 \times 10^{-12}$ & 0.300 & $1.140 \times 10^{-03}$ \\
\hline 0.013 & $3.426 \times 10^{-12}$ & 0.350 & $2.026 \times 10^{-03}$ \\
\hline 0.014 & $7.121 \times 10^{-12}$ & 0.400 & $3.282 \times 10^{-03}$ \\
\hline 0.015 & $1.384 \times 10^{-11}$ & 0.450 & $4.971 \times 10^{-03}$ \\
\hline 0.016 & $2.543 \times 10^{-11}$ & 0.500 & $7.163 \times 10^{-03}$ \\
\hline 0.018 & $7.457 \times 10^{-11}$ & 0.600 & $1.343 \times 10^{-02}$ \\
\hline 0.020 & $1.884 \times 10^{-10}$ & 0.700 & $2.318 \times 10^{-02}$ \\
\hline 0.025 & $1.207 \times 10^{-09}$ & 0.800 & $3.809 \times 10^{-02}$ \\
\hline 0.030 & $4.979 \times 10^{-09}$ & 0.900 & $6.021 \times 10^{-02}$ \\
\hline 0.040 & $3.938 \times 10^{-08}$ & 1.000 & $9.140 \times 10^{-02}$ \\
\hline 0.050 & $1.720 \times 10^{-07}$ & 1.250 & $2.160 \times 10^{-01}$ \\
\hline 0.060 & $5.313 \times 10^{-07}$ & 1.500 & $4.055 \times 10^{-01}$ \\
\hline 0.070 & $1.311 \times 10^{-06}$ & 1.750 & $6.435 \times 10^{-01}$ \\
\hline 0.080 & $2.771 \times 10^{-06}$ & 2.000 & $9.100 \times 10^{-01}$ \\
\hline
\end{tabular}

Therefore, by using the Kontos et al. rate in essence the ${ }^{6} \mathrm{Li} /{ }^{7} \mathrm{Li}$ isotopic ratio is determined by the ratio of two LUNA experimental S-factors in similar setups: The present ${ }^{2} \mathrm{H}(\alpha, \gamma){ }^{6} \mathrm{Li}$ data on ${ }^{6} \mathrm{Li}$ production, and the previous ${ }^{3} \mathrm{He}(\alpha, \gamma){ }^{7} \mathrm{Be}$ data $[35,36]$ on ${ }^{7} \mathrm{Be} \rightarrow{ }^{7} \mathrm{Li}$ production.

Using the Kontos et al. rate [32], ${ }^{7} \mathrm{Li} / \mathrm{H}=(5.2 \pm 0.4) \times 10^{-10}$ is found, $15 \%$ higher than when using CF88. Finally, one obtains a lithium isotopic ratio of ${ }^{6} \mathrm{Li} /{ }^{7} \mathrm{Li}=(1.6 \pm 0.3) \times 10^{-5}$. The ${ }^{6} \mathrm{Li} /{ }^{7} \mathrm{Li}$ error is dominated by the $22 \%$ uncertainty on ${ }^{6} \mathrm{Li}$. The ${ }^{7} \mathrm{Li}$ abundance prediction is known at the $8 \%$ level [37].

Finally, the BBN calculations have then been repeated using the PARTHENOPE (Naples) code [38] instead of SKM. PARTHENOPE features an up to date nuclear reaction network, including neutron induced reactions. No deviations were expected, as the presently discussed $\alpha$ capture reactions strongly dominate production. Indeed, the ${ }^{6} \mathrm{Li} /{ }^{7} \mathrm{Li}$ isotopic ratio from PARTHENOPE is found to be consistent with the SKM result, and SKM is adopted henceforth.

The present ${ }^{6} \mathrm{Li} /{ }^{7} \mathrm{Li}$ ratio of $(1.6 \pm 0.3) \times 10^{-5}$ is lower than but still consistent with - the previous PARTHENOPE value of (2$3) \times 10^{-5}$ that did not make use of the present cross section data [13]. Also, it is lower than the recently published value of $2.3 \times$ $10^{-5}$ [39] that did not yet make use of the present data.

Finally, the present ${ }^{6} \mathrm{Li} /{ }^{7} \mathrm{Li}$ ratio is much lower than obtained from the reported ${ }^{6} \mathrm{Li}$ detections in metal-poor stars $[3,4,10]$ and in the Small Magellanic Cloud [40].

\section{Conclusions}

In this work, we report on the first direct measurement of the ${ }^{2} \mathrm{H}(\alpha, \gamma){ }^{6} \mathrm{Li}$ reaction in the BBN energy range. The new data do not provide a nuclear solution to the "second lithium problem". Therefore, further astronomical investigations and astrophysical modelling are desirable, as well as new cross section measurements in the energy range up to $1 \mathrm{MeV}$. Assuming that observations will confirm a somewhat higher ${ }^{6} \mathrm{Li}$ abundance with respect to the computed $\Lambda \mathrm{CDM}$ (Lambda Cold Dark Matter) model value, 
the only remaining possibilities to explain the discrepancy are very special astrophysical processes like stellar flare in-situ lithium production [41]. Other possibilities are unknown physical processes or new physics scenarios [42-45].

\section{Acknowledgments}

The authors are indebted to F. L. Villante (INFN-LNGS) for informative conversations on BBN calculations, and to the mechanical and electronic workshops of LNGS for technical support. Financial support by INFN, FAI, DFG (Grant No. BE 4100-2/1), NAVI (Grant No. HGF VH-VI-417), and OTKA (Grant No. K101328) is gratefully acknowledged.

\section{References}

[1] Planck Collaboration, P. A. R. Ade, N. Aghanim, M. Arnaud, M. Ashdown, J. Aumont, C. Baccigalupi, A. J. Banday, R. B. Barreiro, J. G. Bartlett, et al., Planck 2015 results. XIII. Cosmological parameters, ArXiv e-prints (2015).

[2] B.D. Fields, The primordial lithium problem, Annu. Rev. Nucl. Part. Sci. 61 (2011) 47-68, doi:10.1146/annurev-nucl-102010-130445.

[3] M. Asplund, D.L. Lambert, P.E. Nissen, F. Primas, V.V. Smith, Lithium isotopic abundances in metal-poor halo stars, Astrophys. J. 644 (2006) 229-259, doi:10. 1086/503538.

[4] M. Asplund, J. Meléndez, Primordial and pre-galactic origins of the Lithium isotopes, in: B.W. O'Shea, A. Heger (Eds.), First Stars III, vol. 990 of AIP Conference Proceedings, 2008, pp. 342-346, doi:10.1063/1.2905578.

[5] M. Anders, D. Trezzi, R. Menegazzo, M. Aliotta, A. Bellini, D. Bemmerer, C. Broggini, A. Caciolli, P. Corvisiero, H. Costantini, T. Davinson, Z. Elekes, M. Erhard, A. Formicola, Z. Fülöp, G. Gervino, A. Guglielmetti, C. Gustavino, G. Gyürky, M. Junker, A. Lemut, M. Marta, C. Mazzocchi, P. Prati, C. Rossi Alvarez, D.A. Scott, E. Somorjai, O. Straniero, T. Szücs, First direct measurement of the ${ }^{2} \mathrm{H}(\alpha, \gamma){ }^{6} \mathrm{Li}$ cross section at big bang energies and the primordial lithium problem, Phys. Rev. Lett. 113 (2014) 042501, doi:10.1103/PhysRevLett.113.042501.

[6] M. Anders, D. Trezzi, A. Bellini, M. Aliotta, D. Bemmerer, C. Broggini, A. Caciolli, H. Costantini, P. Corvisiero, T. Davinson, Z. Elekes, M. Erhard, A. Formicola, Z. Fülöp, G. Gervino, A. Guglielmetti, C. Gustavino, G. Gyürky, M. Junker, A. Lemut, M. Marta, C. Mazzocchi, R. Menegazzo, P. Prati, C. Rossi Alvarez, D. Scott, E. Somorjai, O. Straniero, T. Szücs, Neutron-induced background by an $\alpha$-beam incident on a deuterium gas target and its implications for the study of the ${ }^{2} \mathrm{H}(\alpha, \gamma)^{6} \mathrm{Li}$ reaction at LUNA, Eur. Phys. J. A 49 (2013) 28, doi:10.1140/ epja/i2013-13028-5.

[7] H. Reeves, Galactic cosmic ray origin of Li, Be and B in stars, Nature 226 (1970) 727-729, doi:10.1038/226727a0

[8] V.V. Smith, D.L. Lambert, P.E. Nissen, The ${ }^{6} \mathrm{Li} /{ }^{7} \mathrm{Li}$ ratio in the metal-poor halo dwarfs HD 19445 and HD 84937, Astrophys. J. 408 (1993) 262-276, doi:10. $1086 / 172585$.

[9] R. Cayrel, M. Spite, F. Spite, E. Vangioni-Flam, M. Cassé, J. Audouze, New high S/N observations of the ${ }^{6} \mathrm{Li} /{ }^{7} \mathrm{Li}$ blend in HD 84937 and two other metal-poor stars, Astron. Astrophys. 343 (1999) 923-932.

[10] M. Steffen, R. Cayrel, E. Caffau, P. Bonifacio, H.-G. Ludwig, M. Spite, ${ }^{6}$ Li detection in metal-poor stars: can 3D model atmospheres solve the second lithium problem? Mem. S.A.It. 22 (2012) 152.

[11] K. Lind, J. Melendez, M. Asplund, R. Collet, Z. Magic, The lithium isotopic ratio in very metal-poor stars, Astron. Astrophys. 554 (2013) A96, doi:10.1051/ 0004-6361/201321406

[12] E. Caffau, M. Steffen, P. Bonifacio, H.-G. Ludwig, L. Monaco, G. Lo Curto, I. Kamp, Isotope spectroscopy, Astron. Nachr. 335 (1) (2014) 59-64, doi:10. 1002/asna.201312010.

[13] P.D. Serpico, S. Esposito, F. Iocco, G. Mangano, G. Miele, O. Pisanti, Nuclear reaction network for primordial nucleosynthesis: a detailed analysis of rates, uncertainties and light nuclei yields, J. Cosmol. Astropart. Phys. 2004 (12) (2004) 010.

[14] Y. Xu, K. Takahashi, S. Goriely, M. Arnould, M. Ohta, H. Utsunomiya, NACRE II: an update of the NACRE compilation of charged-particle-induced thermonuclear reaction rates for nuclei with mass number $A<16$, Nucl. Phys. A 918 (2013) 61-169, doi:10.1016/j.nuclphysa.2013.09.007.

[15] F. Hammache, M. Heil, S. Typel, D. Galaviz, K. Sümmerer, A. Coc, F. Uhlig, F. Attallah, M. Caamano, D. Cortina, H. Geissel, M. Hellström, N. Iwasa, J. Kiener, P. Koczon, B. Kohlmeyer, P. Mohr, E. Schwab, K. Schwarz, F. Schümann, P. Senger, O. Sorlin, V. Tatischeff, J.P. Thibaud, E. Vangioni, A. Wagner, W. Walus, High-energy breakup of ${ }^{6} \mathrm{Li}$ as a tool to study the Big Bang nucleosynthesis reaction ${ }^{2} \mathrm{H}(\alpha, \gamma){ }^{6} \mathrm{Li}$, Phys. Rev. C 82 (2010) 065803, doi:10.1103/PhysRevC.82. 065803.

[16] K.M. Nollett, Radiative $\alpha$-capture cross sections from realistic nucleon-nucleon interactions and variational Monte Carlo wave functions, Phys. Rev. C 63 (2001) 054002, doi:10.1103/PhysRevC.63.054002.

[17] F.E. Cecil, J. Yan, C.S. Galovich, The reaction $\mathrm{d}(\alpha, \gamma)^{6} \mathrm{Li}$ at low energies and the primordial nucleosynthesis of ${ }^{6} \mathrm{Li}$, Phys. Rev. C 53 (1996) 1967-1970, doi:10. 1103/PhysRevC.53.1967.
[18] P. Mohr, V. Kölle, S. Wilmes, U. Atzrott, G. Staudt, J.W. Hammer, H. Krauss $\mathrm{H}$. Oberhummer, Direct capture in the $3^{+}$resonance of ${ }^{2} \mathrm{H}(\alpha, \gamma)^{6} \mathrm{Li}$, Phys. Rev. C 50 (1994) 1543-1549, doi:10.1103/PhysRevC.50.1543.

[19] R.G.H. Robertson, et al., Observation of the capture reaction ${ }^{2} \mathrm{H}(\alpha, \gamma){ }^{6} \mathrm{Li}$ and its role in production of ${ }^{6} \mathrm{Li}$ in the big bang, Phys. Rev. Lett. 47 (1981) 1867-1870.

[20] J. Kiener, HJ Gils, H. Rebel, S. Zagromski, G. Gsottschneider, N. Heide, H. Jelitto, J. Wentz, G. Baur, Measurements of the Coulomb dissociation cross section of $156 \mathrm{MeV}{ }^{6} \mathrm{Li}$ projectiles at extremely low relative fragment energies of astrophysical interest, Phys. Rev. C 44 (1991) 2195-2208.

[21] A. Formicola, G. Imbriani, M. Junker, D. Bemmerer, R. Bonetti, C. Broggini, C. Casella, P. Corvisiero, H. Costantini, G. Gervino, C. Gustavino, A. Lemut, P. Prati, V. Roca, C. Rolfs, M. Romano, D. Schürmann, F. Strieder, F. Terrasi, H.P. Trautvetter, S. Zavatarelli, The LUNA II $400 \mathrm{kV}$ accelerator, Nucl. Inst. Meth. A 507 (2003) 609-616, doi:10.1016/S0168-9002(03)01435-9.

[22] M. Anders, S-factor Measurement of the ${ }^{2} \mathrm{H}(\alpha, \gamma){ }^{6}$ Li Reaction at Energies Relevant for Big-Bang Nucleosynthesis, Technical University of Dresden, 2013 Report HZDR-042 (2013) and PhD Thesis.

[23] M.A. Erhard, Photoaktivierung des p-Kerns Mo-92 am Bremsstrahlungsmessplatz von ELBE, Technical University of Dresden, 2010 PhD Thesis.

[24] M. Erhard, E. Grosse, A. Hartmann, A.R. Junghans, C. Nair, K.D. Schilling, W. Schulze, R. Schwengner, The photoactivation setup at ELBE, in: W. Enghardt, K. Fahmy, E. Grosse, B. Kämpfer, C. Schneidereit, A. Wagner (Eds.), Annual Report 2004, Institute of Nuclear and Hadron Physics, FZR-423, Forschungszentrum Rossendorf, 2005, p. 22.

[25] C. Rolfs, W. Rodney, Cauldrons in the Cosmos, University of Chicago Press, Chicago, 1988

[26] C. Gustavino, LUNA data on the ${ }^{2} \mathrm{H}(\alpha, \gamma){ }^{6} \mathrm{Li}$ reaction and Big Bang nucleosynthesis, in: XII International Symposium on Nuclei in the Cosmos, PoS(NIC XII)074, 2012.

[27] A.M. Mukhamedzhanov, L.D. Blokhintsev, B.F. Irgaziev, Reexamination of the astrophysical s factor for the $\alpha+\mathrm{d} \rightarrow{ }^{6} \mathrm{Li}+\gamma$ reaction, Phys. Rev. C 83 (5) (2011) 055805, doi:10.1103/PhysRevC.83.055805

[28] G. Caughlan, W. Fowler, Thermonuclear reaction rates V, At. Data Nucl. Data Tables 40 (1988) 283-334.

[29] C. Angulo, M. Arnould, M. Rayet, P. Descouvemont, D. Baye, C. Leclercq-Willain, A. Coc, S. Barhoumi, P. Aguer, C. Rolfs, R. Kunz, J.W. Hammer, A. Mayer, T. Paradellis, S. Kossionides, C. Chronidou, K. Spyrou, S. degl'Innocenti, G. Fiorentini, B. Ricci, S. Zavatarelli, C. Providencia, H. Wolters, J. Soares, C. Grama, J. Rahighi, A. Shotter, M. Lamehi Rachti, A compilation of charged-particle induced thermonuclear reaction rates, Nucl. Phys. A 656 (1999) 3-187.

[30] M.S. Smith, L.H. Kawano, R.A. Malaney, Experimental, computational, and observational analysis of primordial nucleosynthesis, Astrophys. J. Suppl. Ser. 85 (1993) 219-247, doi:10.1086/191763.

[31] K.A. Olive, Particle Data Group, Review of particle physics, Chin. Phys. C 38 (9) (2014) 090001, doi:10.1088/1674-1137/38/9/090001.

[32] A. Kontos, E. Uberseder, R. deBoer, J. Görres, C. Akers, A. Best, M. Couder, M. Wiescher, Astrophysical S factor of ${ }^{3} \mathrm{He}(\alpha, \gamma)^{7}$ Be, Phys. Rev. C 87 (6) (2013) 065804, doi:10.1103/PhysRevC.87.065804.

[33] R.J. deBoer, J. Görres, K. Smith, E. Uberseder, M. Wiescher, A. Kontos, G. Imbriani, A. Di Leva, F. Strieder, Monte Carlo uncertainty of the ${ }^{3} \mathrm{He}(\alpha, \gamma)^{7} \mathrm{Be}$ reaction rate, Phys. Rev. C 90 (2014) 035804, doi:10.1103/PhysRevC.90.035804.

34] M.P. Takács, D. Bemmerer, T. Szücs, K. Zuber, Constraining Big Bang lithium production with recent solar neutrino data, Phys. Rev. D 91 (2015) 123526, doi:10.1103/PhysRevD.91.123526.

[35] D. Bemmerer, F. Confortola, H. Costantini, A. Formicola, G. Gyürky, R. Bonetti, C. Broggini, P. Corvisiero, Z. Elekes, Z. Fülöp, G. Gervino, A. Guglielmetti, C. Gustavino, G. Imbriani, M. Junker, M. Laubenstein, A. Lemut, B. Limata, V. Lozza, M. Marta, R. Menegazzo, P. Prati, V. Roca, C. Rolfs, C.R. Alvarez, E. Somorjai, O. Straniero, F. Strieder, F. Terrasi, H.P. Trautvetter, Activation measurement of the ${ }^{3} \mathrm{He}(\alpha, \gamma)^{7} \mathrm{Be}$ cross section at low energy, Phys. Rev. Lett. 97 (12) (2006) 122502, doi:10.1103/PhysRevLett.97.122502.

[36] F. Confortola, D. Bemmerer, H. Costantini, A. Formicola, G. Gyürky, P. Bezzon, R. Bonetti, C. Broggini, P. Corvisiero, Z. Elekes, Z. Fülöp, G. Gervino, A. Guglielmetti, C. Gustavino, G. Imbriani, M. Junker, M. Laubenstein, A. Lemut, B. Limata, V. Lozza, M. Marta, R. Menegazzo, P. Prati, V. Roca, C. Rolfs, C.R. Alvarez, E. Somorjai, O. Straniero, F. Strieder, F. Terrasi, H.P. Trautvetter, Astrophysical s factor of the $\mathrm{He} 3(\alpha, \gamma) \mathrm{Be} 7$ reaction measured at low energy via detection of prompt and delayed $\gamma$ rays, Phys. Rev. C 75 (6) (2007) 065803 , doi:10.1103/PhysRevC.75.065803.

[37] A. Coc, J.-P. Uzan, E. Vangioni, Standard big bang nucleosynthesis and primordial CNO abundances after planck, J. Cosmol. Astropart. Phys. 10 (2014) 050, doi:10.1088/1475-7516/2014/10/050.

[38] O. Pisanti, A. Cirillo, S. Esposito, F. Iocco, G. Mangano, G. Miele, P. Serpico, Parthenope: public algorithm evaluating the nucleosynthesis of primordial elements, Computer Phys. Comm. 178 (12) (2008) 956- 971. http://dx.doi.org/10. 1016/j.cpc.2008.02.015

[39] A. Coc, S. Goriely, Y. Xu, M. Saimpert, E. Vangioni, Standard big bang nucleosynthesis up to CNO with an improved extended nuclear network, Astrophys. J. 744 (2012) 158, doi:10.1088/0004-637X/744/2/158.

[40] J.C. Howk, N. Lehner, B.D. Fields, G.J. Mathews, Observation of interstellar lithium in the low-metallicity small magellanic cloud, Nature 489 (2012) 121123, doi:10.1038/nature11407.

[41] N. Prantzos, Production and evolution of Li, Be, and B isotopes in the galaxy, Astron. Astrophys. 542 (2012) A67, doi:10.1051/0004-6361/201219043.

[42] K. Jedamzik, M. Pospelov, Big bang nucleosynthesis and particle dark matter, New J. Phys. 11 (2009) 105028, doi:10.1088/1367-2630/11/10/105028. 
[43] M. Pospelov, J. Pradler, Big bang nucleosynthesis as a probe of new physics, Annu. Rev. Nucl. Part. Sci. 60 (2010) 539-568, doi:10.1146/annurev.nucl.012809. 104521.

[44] H. Djapo, I. Boztosun, G. Kocak, A.B. Balantekin, Influence of long-range effects on low-energy cross sections of He and Hex: the lithium problem, Phys. Rev. C 85 (2012) 044602, doi:10.1103/PhysRevC.85.044602.
[45] M. Kusakabe, T. Kajino, R.N. Boyd, T. Yoshida, G.J. Mathews, Simultaneous solution to the ${ }^{6} \mathrm{Li}$ and ${ }^{7} \mathrm{Li}$ big bang nucleosynthesis problems from a longlived negatively charged leptonic particle, Phys. Rev. D 76 (2007) 121302, doi:10.1103/PhysRevD.76.121302. 